УДК 517.953

\title{
Unified Transform method for the Schrödinger Equation on a Simple Metric Graph
}

\author{
Gulmirza Khudayberganov* \\ Zarifboy A. Sobirov ${ }^{\dagger}$ \\ Mardonbek R. Eshimbetov ${ }^{\ddagger}$ \\ National University of Uzbekistan \\ Universitet Str. 4, Tashkent, 100174 \\ Uzbekistan
}

Received 16.11.2018, received in revised form 06.02.2019, accepted 05.04.2019

Integral-representation of solutions of the initial-boundary value problems for the Schrödinger equation on simple metric graphs was obtained with the use of the Fokas method. This method uses special generalization of the Fourier transform that is referred to as the unified transform. Obtained representation of solutions of the problem for open and closed simple star graphs allows one to identify transmitted, reflected and trapped waves at the graph branching point.

Keywords: Schrödinger equation, metric graph, branched structure, unified transform, initial problem, Fourier transform, boundary value problem.

DOI: $10.17516 / 1997-1397-2019-12-4-412-420$.

\section{Introduction}

It is known that branched structures and networks are used to model many complex systems from physics, biology, ecology, sociology, economy and finance [1,2]. In this paper we present the Fokas method for the solution of time dependent Schrödinger equation on simple star graphs. Metric graphs with Schrödinger equation accompanied together with Kirchhoff conditions at the vertex are referred to as quantum graphs [3-5]. Usually static Schrödinger equation is used to study physical properties of quantum graphs (see [3-7] and references therein). Non linear Schrödinger equation on two dimensional thin tabular branched domain was studied [8]. It was proved that the problem on metric graphs for one dimensional non linear Schrödinger equation on metric graph with gluing (Kirchhoff) conditions at the vertex can be obtained when width of the branches tends to zero. Similar convergence result in the case of linear Schrödinger equation with different approaches was obtained $[6,7]$.

Schrödinger equation can be also treated as the equation with imaginary time. The heat equation on branched structures was firstly used in the 50's of the nineteenth century. Thomson (Lord Kelvin) used the heat equation (Thomson's cable equation) as mathematical model of signal decay in submarine (underwater) telegraphic cables (Ch. IV in [9]). Later this method was widely used in neuroscience to analyse data collected from intracellular microelectrode recordings and to analyse the electrical properties of neuronal dendrites (see [10,11]). Initial and boundary value problems for some other types of PDE on metric graphs and their possible applications can be found in $[12-14]$.

In this paper we consider time-dependent Schrödinger equation. To construct solution of Schrödinger equation on simple star graphs with bounded and semi-infinite bonds we use the

\footnotetext{
*gkhudaiberg@mail.ru

†sobirovzar@gmail.com

$\ddagger$ mr.eshimbetov92@mail.ru

(c) Siberian Federal University. All rights reserved
} 
Fokas unified transform method [15-19]. Our solution gives more detailed information on scattering at the branching point of the graph and dynamics near vertex.

\section{Simple star graph with semi-infinite bonds}

Let us consider simple star graph $\Gamma_{1}$ with three semi-infinite bonds connected at the point $O$. The point $O$ is the vertex of the graph. We label bonds of the graph as $B_{j}, j=1,2,3$ (Fig. 1). Let us define coordinate $x_{j}$ on the bond $B_{j}, j=1,2,3$, and $x_{j} \in(0, \infty)$. We denote the graph by $\Gamma_{\infty}$. At each bond the coordinate of the vertex point $O$ is equal to zero. Further we will use $x$ instead of $x_{j}$.

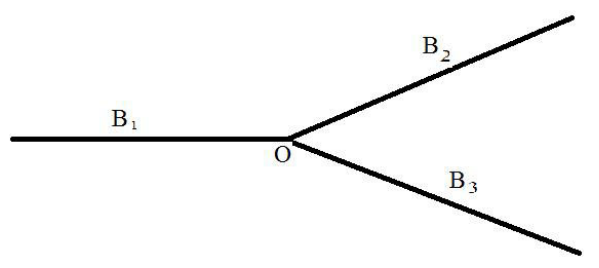

Fig. 1

In each bond of the graph we consider Schrödinger equation

$$
i q_{t}^{(j)}(x, t)=\sigma q_{x x}^{(j)}(x, t), \quad x \in B_{j}, \quad t>0, \quad j=1,2,3,
$$

where $\sigma=\frac{-\hbar}{2 m}$. Initial conditions are

$$
q^{(j)}(x, 0)=q_{0}^{(j)}(x), \quad x \in B_{j}, \quad j=1,2,3,
$$

and asymptotic conditions are

$$
\lim _{x \rightarrow \infty} q^{(j)}(x, t)=0, \quad t \geqslant 0, \quad j=1,2,3 .
$$

At the vertex point the solution satisfies the following gluing (Kirchhoff) conditions

$$
\begin{gathered}
q^{(1)}(+0, t)=q^{(2)}(+0, t)=q^{(3)}(+0, t), \quad t \geqslant 0, \\
\delta_{1}^{2} q_{x}^{(1)}(+0, t)+\delta_{2}^{2} q_{x}^{(2)}(+0, t)+\delta_{3}^{2} q_{x}^{(3)}(+0, t)=0, \quad t \geqslant 0 .
\end{gathered}
$$

Now we consider standard steps in the application of the Fokas method. We begin with the so-called "local relations" (see [17]):

$$
\left(e^{-i k x+w t} q^{(j)}(x, t)\right)_{t}=\left(\sigma e^{-i k x+w t}\left(k q^{(j)}(x, t)-i q_{x}^{(j)}(x, t)\right)\right)_{x}, \quad j=1,2,3
$$

where $w(k)=-i \sigma k^{2}$.

Integrating over domain $\Gamma_{\infty} \times(0, t)$ and applying Green's theorem, we find (Fig. 2)

$$
\begin{aligned}
0=\int_{0}^{\infty} e^{-i k x} q_{0}^{(j)}(x) d x-\int_{0}^{\infty} & e^{-i k x+w t} q^{(j)}(x, t) d x- \\
& -\int_{0}^{t} \sigma e^{w s}\left(k q^{(j)}(0, s)-i q_{x}^{(j)}(0, s)\right) d s, \quad j=1,2,3,
\end{aligned}
$$

where $k \in \mathbb{C}$. 


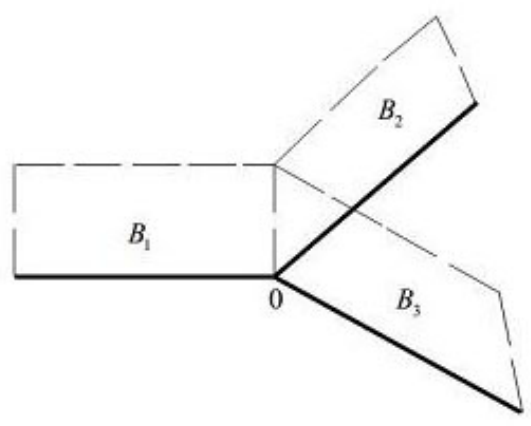

Fig. 2

One can notice that this procedure is equivalent to application of the Fourier transform in (1) with respect to time and coordinate. But in this Green's theorem we use special dispersion relation $w=-i \sigma k^{2}$ mentioned above. So, in this Fourier transform parameters $w$ and $k$ are not independent. Relation between these parameters (or new variables) should be chosen from the following equations

$$
\begin{gathered}
\widehat{q}^{(j)}(k, t)=\int_{0}^{\infty} e^{-i k x} q^{(j)}(x, t) d x, \quad j=1,2,3, \\
\widehat{q}_{0}^{(j)}(k)=\int_{0}^{\infty} e^{-i k x} q_{0}^{(j)}(x) d x, \quad j=1,2,3 .
\end{gathered}
$$

Using these definitions, the global relation (7) is rewritten as

$$
e^{w t} \widehat{q}^{(j)}(k, t)=\widehat{q}_{0}^{(j)}(k)-k \sigma g_{0}(w, t)+i \sigma g_{j}(w, t), \quad j=1,2,3,
$$

where $\{k \in \mathbb{C}: \operatorname{Im} k>0\}, j=1,2,3$.

Since the dispersion relation $w=-i \sigma k^{2}$ is invariant with respect to transform $k \rightarrow-k$ then functions $g_{0}(w, t), g_{j}(w, t), j=1,2,3$ are also invariant with respect to this transform. Thus we can substitute $-k$ instead of $k$ into (8) and obtain

$$
e^{w t} \widehat{q}^{(j)}(-k, t)=\widehat{q}_{0}^{(j)}(-k)+k \sigma g_{0}(w, t)+i \sigma g_{j}(w, t), \quad j=1,2,3
$$

where $\{k \in \mathbb{C}: \operatorname{Im} k<0\}, j=1,2,3$.

Inverting the Fourier transforms in (8), we have

$$
\begin{aligned}
q^{(j)}(x, t)=\frac{1}{2 \pi} \int_{-\infty}^{\infty} e^{i k x-w t} \widehat{q}_{0}^{(j)}(k) d k+ & \\
& +\frac{1}{2 \pi} \int_{-\infty}^{\infty} e^{i k x-w t}\left(-k \sigma g_{0}(w, t)+i \sigma g_{j}(w, t)\right) d k, j=1,2,3 .
\end{aligned}
$$

The region $D$ is shown in Fig. 3. The integrand of the second integral in (10) is entire and decays as $k \rightarrow \infty$ for $k \in\{I m k>0\} \backslash D^{(2)}$. Using the analyticity of the integrand and applying Jordan's lemma, we can replace the contour of integration of the second integral (see [17-19]):

$$
\begin{aligned}
q^{(j)}(x, t)=\frac{1}{2 \pi} \int_{-\infty}^{\infty} e^{i k x-w t} \widehat{q}_{0}^{(j)}(k) d k+ & \\
& +\frac{1}{2 \pi} \int_{\partial D^{(2)}} e^{i k x-w t}\left(-k \sigma g_{0}(w, t)+i \sigma g_{j}(w, t)\right) d k, \quad j=1,2,3,
\end{aligned}
$$

where $D=\left\{k \in \mathbb{C}: \operatorname{Re}\left(-i k^{2}\right)<0\right\}=D^{(2)} \bigcup D^{(4)}$. 


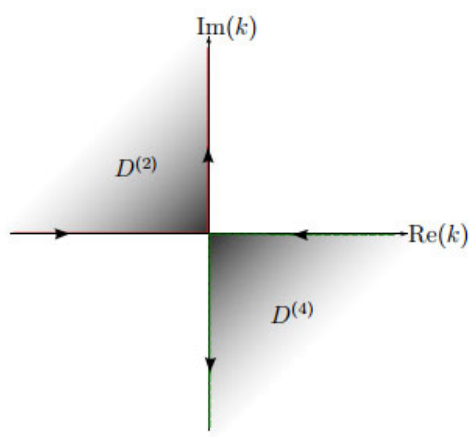

Fig. 3

We need to find unknowns $g_{j}(w, t), g_{0}(w, t), j=1,2,3$ in this representation of the solution. Solving (8) for $i \sigma g_{j}(w, t)$, we find

$$
i \sigma g_{j}(w, t)=e^{w t} \widehat{q}^{(j)}(-k, t)-\widehat{q}_{0}^{(j)}(-k)-k \sigma g_{0}(w, t), \quad I m k \geqslant 0 .
$$

So, we have

$$
\begin{aligned}
q^{(j)}(x, t)= & \frac{1}{2 \pi} \int_{-\infty}^{+\infty} e^{i k x-w t} \widehat{q}_{0}^{(j)}(k) d k- \\
& -\frac{1}{2 \pi} \int_{\partial D^{(2)}} e^{i k x} \widehat{q}^{(j)}(-k, t) d k \\
& +\frac{1}{2 \pi} \int_{\partial D^{(2)}} e^{i k x-w t} \widehat{q}_{0}^{(j)}(-k) d k- \\
& -\frac{1}{\pi} \int_{\partial D^{(2)}} e^{i k x-w t} k \sigma g_{0}(w, t) d k, \quad j=1,2,3 .
\end{aligned}
$$

Taking to account that functions $e^{i k x}$ and $\widehat{q}^{(j)}(-k, t)$ are holomorphic and bounded in $\{k \in \mathbb{C}: \operatorname{Im} k>0\}$, we conclude that the following integral (see [17-19])

$$
\frac{1}{2 \pi} \int_{\partial D^{(2)}} e^{i k x} \widehat{q}^{(j)}(-k, t) d k, \quad 0<x<\infty, \quad t>0
$$

tends to zero at $k \rightarrow \infty$.

The term $e^{i k x} \widehat{q}^{(j)}(-k, t)$ gives rise to the term

$$
\frac{1}{2 \pi} \int_{\partial D^{(2)}} e^{i k x} \widehat{q}^{(j)}(-k, t) d k, \quad 0<x<\infty, \quad t>0
$$

which vanishes because both $e^{i k x}$ and $\widehat{q}^{(j)}(-k, t)$ are bounded and analytic in the upper half of the complex $k$ plane, and furthermore $\widehat{q}^{(j)}(k, t)$ is of $O\left(\frac{1}{k}\right)$ as $k \rightarrow \infty$ :

$$
\widehat{q}^{(j)}(k, t)=\int_{0}^{\infty} e^{i k x} q^{(j)}(x, t) d x \sim \frac{q^{(j)}(0, t)}{k}, \quad k \rightarrow \infty .
$$

Thus, Cauchy's theorem supplemented with Jordan's lemma in the domain $D^{(2)}$ implies the desired result.

Hence

$$
\begin{aligned}
q^{(j)}(x, t)=\frac{1}{2 \pi} \int_{-\infty}^{+\infty} e^{i k x-w t} \widehat{q}_{0}^{(j)}(k) d k & +\frac{1}{2 \pi} \int_{\partial D^{(2)}} e^{i k x-w t} \widehat{q}_{0}^{(j)}(-k) d k- \\
& -\frac{1}{\pi} \int_{\partial D^{(2)}} e^{i k x-w t} k \sigma g_{0}(w, t) d k, \quad j=1,2,3 . \\
& -415-
\end{aligned}
$$


Using vertex conditions, we obtain

$$
\left\{\begin{array}{l}
e^{w t} \widehat{q}^{(1)}(-k, t)=\widehat{q}_{0}^{(1)}(-k)+k \sigma g_{0}(w, t)+i \sigma g_{1}(w, t) \\
e^{w t} \widehat{q}^{(2)}(-k, t)=\widehat{q}_{0}^{(2)}(-k)+k \sigma g_{0}(w, t)+i \sigma g_{2}(w, t) \\
e^{w t} \widehat{q}^{(3)}(-k, t)=\widehat{q}_{0}^{(3)}(-k)+k \sigma g_{0}(w, t)+i \sigma g_{3}(w, t) \\
\delta_{1}^{2} q_{x}^{(1)}(+0, t)+\delta_{2}^{2} q_{x}^{(2)}(+0, t)+\delta_{3}^{2} q_{x}^{(3)}(+0, t)=0
\end{array} .\right.
$$

where $\{k \in \mathbb{C}: \operatorname{Im} k<0\}$. Solving this equations for $g_{0}$, we have

$$
\begin{gathered}
k \sigma g_{0}(w, t)=-\frac{\delta_{1}^{2} \widehat{q}_{0}^{(1)}(-k)+\delta_{2}^{2} \widehat{q}_{0}^{(2)}(-k)+\delta_{3}^{2} \widehat{q}_{0}^{(3)}(-k)}{\delta_{1}^{2}+\delta_{2}^{2}+\delta_{3}^{2}}+ \\
+\frac{e^{w t}\left(\delta_{1}^{2} \widehat{q}^{(1)}(-k, t)+\delta_{2}^{2} \widehat{q}^{(2)}(-k, t)+\delta_{3}^{2} \widehat{q}^{(3)}(-k, t)\right)}{\delta_{1}^{2}+\delta_{2}^{2}+\delta_{3}^{2}}
\end{gathered}
$$

Substituting (16) into (14) and taking to account that some integrals vanish, we obtain the solution of the problem

$$
\begin{aligned}
q^{(j)}(x, t)=\frac{1}{2 \pi} \int_{-\infty}^{+\infty} e^{i k x-w t} \widehat{q}_{0}^{(j)}(k) d k+ & \\
+ & \frac{1}{2 \pi} \int_{\partial D^{(2)}} e^{i k x-w t} \frac{\delta_{1}^{2}-\delta_{2}^{2}-\delta_{3}^{2}}{\delta_{1}^{2}+\delta_{2}^{2}+\delta_{3}^{2}} \widehat{q}_{0}^{(j)}(-k) d k+ \\
& +\frac{1}{\pi} \int_{\partial D^{(2)}} e^{i k x-w t} \frac{\delta_{2}^{2} \widehat{q}_{0}^{(2)}(-k)+\delta_{3}^{2} \widehat{q}_{0}^{(3)}(-k)}{\delta_{1}^{2}+\delta_{2}^{2}+\delta_{3}^{2}} d k, \quad j=1,2,3 .
\end{aligned}
$$

First term in this solution represents free wave propagation. Second and third terms represent reflection and transmission to the other bonds.

In conclusion we have

Theorem 1. If initial data $q_{0}^{(j)}(x)$ are analitical functions in $\bar{B}_{j}$ for $j=1,2,3$, then the solution of problem (1)-(6) is given by (17).

\section{Simple star graph with bounded bonds}

Here we consider simple graph $\Gamma_{2}$ with three bounded bonds connected at the point $O$. The point $O$ is the vertex of the graph. Let us denote bonds of the graph by $B_{j}, j=1,2,3$. We define coordinate $x_{1} \in\left(0, L_{1}\right)$ on the bond $B_{1}$. We also define coordinates $x_{2} \in\left(0, L_{2}\right)$ and $x_{3} \in\left(0, L_{3}\right)$ on the bonds $B_{2}$ and $B_{3}$, respectively. At each bond the coordinate of the vertex point $O$ is equal to zero. We denote this graph by $\Gamma$.

We define Schrödinger equations on each bond

$$
i q_{t}^{(j)}(x, t)=\sigma q_{x x}^{(j)}(x, t), \quad x \in B_{j}, \quad t>0, \quad j=1,2,3
$$

with initial conditions

$$
q^{(j)}(x, 0)=q_{0}^{(j)}(x), \quad x \in B_{j}, \quad j=1,2,3
$$

and boundary conditions

$$
q^{(j)}\left(L_{j}, t\right)=h_{0}^{(j)}(t), \quad t \geqslant 0, \quad j=1,2,3
$$

We suppose that solution satisfies the Kirchhoff conditions (4) and (5) at the vertex point. 
Global relations for this case have the form

$$
e^{w t} \widehat{q}^{(j)}(k, t)-\widehat{q}_{0}^{(j)}(k)=e^{-i k L_{j}} \sigma\left(k h_{0}^{(j)}(w, t)-i h_{1}^{(j)}(w, t)\right)-\sigma k g_{0}(w, t)+i \sigma g_{j}(w, t),
$$

where $\{k \in \mathbb{C}: \operatorname{Im} k>0\}, j=1,2,3$,

$$
\begin{gathered}
\widehat{q}^{(j)}(k, t)=\int_{0}^{L_{j}} e^{-i k x} q^{(j)}(x, t) d x ; \widehat{q}_{0}^{(j)}(k)=\int_{0}^{L_{j}} e^{-i k x} q_{0}^{(j)}(x) d x, \\
h_{1}^{(j)}(w, t)=\int_{0}^{t} e^{w s} q_{x}^{(j)}\left(L_{j}, s\right) d s ; h_{0}^{(j)}(w, t)=\int_{0}^{t} e^{w s} q^{(j)}\left(L_{j}, s\right) d s, \\
g_{j}(w, t)=\int_{0}^{t} e^{w s} q_{x}^{(j)}(0, s) d s ; g_{0}(w, t)=\int_{0}^{t} e^{w s} q^{(j)}(0, s) d s, \quad j=1,2,3 .
\end{gathered}
$$

Since the dispersion relation $w=-i \sigma k^{2}$ is invariant with respect to transform $k \rightarrow-k$ then functions $g_{0}(w, t), g_{j}(w, t), h_{1}^{(j)}(w, t), h_{0}^{(j)}(w, t), j=1,2,3$ are also invariant with respect to this transform. Thus we can substitute $-k$ instead of $k$ into (21) and obtain

$$
e^{w t} \widehat{q}^{(j)}(-k, t)-\widehat{q}_{0}^{(j)}(-k)=e^{i k L_{j}} \sigma\left(-k h_{0}^{(j)}(w, t)-i h_{1}^{(j)}(w, t)\right)+\sigma k g_{0}(w, t)+i \sigma g_{j}(w, t),
$$

where $\{k \in \mathbb{C}: \operatorname{Im} k<0\}, j=1,2,3$.

From global relations (21), (22) and the vertex condition

$$
\delta_{1}^{2} g_{1}(w, t)+\delta_{2}^{2} g_{2}(w, t)+\delta_{3}^{2} g_{3}(w, t)=0
$$

we obtain

$$
\begin{aligned}
k \sigma g_{0}(w, t)= & \frac{1}{\delta_{1}^{2} B_{1} A_{2} A_{3}+\delta_{2}^{2} B_{2} A_{1} A_{3}+\delta_{3}^{2} B_{3} A_{1} A_{2}} \times \\
\times & \left(\delta _ { 1 } ^ { 2 } A _ { 2 } A _ { 3 } \left(e^{i k L_{1}} \widehat{q}_{0}^{(1)}(k)-e^{-i k L_{1}} \widehat{q}_{0}^{(1)}(-k)+2 k \sigma h_{0}^{(1)}(w, t)-\right.\right. \\
& \left.-e^{w t}\left(e^{i k L_{1}} \widehat{q}^{(1)}(k, t)-e^{-i k L_{1}} \widehat{q}^{(1)}(-k, t)\right)\right)+ \\
+ & \delta_{2}^{2} A_{1} A_{3}\left(e^{i k L_{2}} \widehat{q}_{0}^{(2)}(k)-e^{-i k L_{2}} \widehat{q}_{0}^{(2)}(-k)+2 k \sigma h_{0}^{(2)}(w, t)-\right. \\
& \left.-e^{w t}\left(e^{i k L_{2}} \widehat{q}^{(2)}(k, t)-e^{-i k L_{2}} \widehat{q}^{(2)}(-k, t)\right)\right)+ \\
+ & \delta_{3}^{2} A_{1} A_{2}\left(e^{i k L_{3}} \widehat{q}_{0}^{(3)}(k)-e^{-i k L_{3}} \widehat{q}_{0}^{(3)}(-k)+2 k \sigma h_{0}^{(3)}(w, t)-\right. \\
& \left.\left.-e^{w t}\left(e^{i k L_{3}} \widehat{q}^{(3)}(k, t)-e^{-i k L_{3}} \widehat{q}^{(3)}(-k, t)\right)\right)\right)
\end{aligned}
$$

where $A_{j}=e^{i k L_{j}}-e^{-i k L_{j}}, B_{j}=e^{i k L_{j}}+e^{-i k L_{j}}, j=1,2,3$.

Introducing $G^{(j)}(k, t)=\widehat{q}_{0}^{(j)}(k)-k \sigma g_{0}(w, t)+e^{-i k L_{j}} \cdot k \sigma h_{0}^{(j)}(w, t), j=1,2,3$, we can rewrite

$$
\left\{\begin{array}{l}
e^{w t} \widehat{q}^{(j)}(k, t)=G^{(j)}(k, t)+i \sigma g_{j}(w, t)-i e^{-i k L_{j}} \sigma h_{1}^{(j)}(w, t), \\
e^{w t} \widehat{q}^{(j)}(-k, t)=G^{(j)}(-k, t)+i \sigma g_{j}(w, t)-i e^{i k L_{j}} \sigma h_{1}^{(j)}(w, t) .
\end{array}\right.
$$

So, we have

$$
\begin{aligned}
& i \sigma g_{j}(w, t)=- \frac{1}{A_{j}}\left(e^{i k L_{j}} G^{(j)}(k, t)-e^{-i k L_{j}} G^{(j)}(-k, t)\right)+ \\
&+\frac{1}{A_{j}}\left(e^{w t}\left(e^{i k L_{j}} \widehat{q}^{(j)}(k, t)-e^{-i k L_{j}} \widehat{q}^{(j)}(-k, t)\right)\right), \\
&-417-
\end{aligned}
$$




$$
\begin{aligned}
i \sigma h_{1}^{(j)}(w, t)=- & \frac{1}{A_{j}}\left(G^{(j)}(k, t)-G^{(j)}(-k, t)\right)+ \\
& +\frac{1}{A_{j}}\left(e^{w t}\left(\widehat{q}^{(j)}(k, t)-\widehat{q}^{(j)}(-k, t)\right)\right), j=1,2,3 .
\end{aligned}
$$

As in the case of the first problem we can state

Theorem 2. The solution of problem (18)-(20), (4), (5) is given by

$$
\begin{aligned}
& q^{(j)}(x, t)=\frac{1}{2 \pi} \int_{-\infty}^{+\infty} e^{i k x-w t} \widehat{q}_{0}^{(j)}(k) d k- \\
& -\frac{1}{2 \pi} \int_{\partial D^{(4)}} e^{i k x-i k L_{j}-w t} \frac{\widehat{q}_{0}^{(j)}(k)-\widehat{q}_{0}^{(j)}(-k)-2 k \sigma g_{0}(w, t)+2 e^{i k L_{j}} k \sigma h_{0}^{(j)}(w, t)}{A_{j}} d k+ \\
& +\frac{1}{2 \pi} \int_{\partial D^{(2)}} e^{i k x-w t} \frac{e^{-i k L_{j}} \widehat{q}_{0}^{(j)}(-k)-e^{i k L_{j}} \widehat{q}_{0}^{(j)}(k)-2 k \sigma h_{0}^{(j)}(w, t)+2 e^{-i k L_{j}} k \sigma g_{0}(w, t)}{A_{j}} d k
\end{aligned}
$$

where $j=1,2,3, g_{0}(w, t)$ is defined by $(23)$.

\section{The general star graph}

Here we generalize the obtained above results to the case of more general star graph which has number of finite and semi-infinite bonds. We consider metric graph $\Gamma_{3}$ which obtained by connecting $n$ finite $B_{1}, B_{2}, \ldots, B_{n}$ and $m$ semi infinite $B_{n+1}, B_{n+2}, \ldots, B_{n+m}$ bonds at one point that is the vertex of the graph. As in previous cases, bonds $B_{j},(j=\overline{1, n})$ correspond to intervals $\left(0, L_{j}\right)$ and bonds $B_{r},(r=\overline{n+1, n+m})$ correspond to intervals $(0, \infty)$. It defines coordinates in each bond.

Theorem 3. The solution of the IBVP problem on $\Gamma_{3}$ has the form

$$
\begin{aligned}
& q^{(j)}(x, t)=\frac{1}{2 \pi} \int_{-\infty}^{+\infty} e^{i k x-w t} \widehat{q}_{0}^{(j)}(k) d k- \\
& -\frac{1}{2 \pi} \int_{\partial D^{(4)}} e^{i k x-i k L_{j}-w t} \frac{\widehat{q}_{0}^{(j)}(k)-\widehat{q}_{0}^{(j)}(-k)-2 k \sigma \widetilde{g}_{0}(w, t)+2 e^{i k L_{j}} k \sigma h_{0}^{(j)}(w, t)}{A_{j}} d k+ \\
& +\frac{1}{2 \pi} \int_{\partial D^{(2)}} e^{i k x-w t} \frac{e^{-i k L_{j}} \widehat{q}_{0}^{(j)}(-k)-e^{i k L_{j}} \widehat{q}_{0}^{(j)}(k)-2 k \sigma h_{0}^{(j)}(w, t)+2 e^{-i k L_{j}} k \sigma \widetilde{g}_{0}(w, t)}{A_{j}} d k \\
& q^{(r)}(x, t)=\frac{1}{2 \pi} \int_{-\infty}^{+\infty} e^{i k x-w t} \widehat{q}_{0}^{(r)}(k) d k+\frac{1}{2 \pi} \int_{\partial D^{(2)}} e^{i k x-w t} \widehat{q}_{0}^{(r)}(-k) d k- \\
& -\frac{1}{\pi} \int_{\partial D^{(2)}} e^{i k x-w t} k \widetilde{g}_{0}(w, t) d k,(j=\overline{1, n}, r=\overline{n+1, n+m)}
\end{aligned}
$$

where

$$
\begin{aligned}
& k \sigma \widetilde{g}_{0}(w, t)=\frac{1}{\sum_{j=1}^{n} \delta_{j}^{2} \frac{B_{j}}{A_{j}}+\sum_{r=n+1}^{n+m} \delta_{r}^{2}} \times \\
& \times\left[\sum_{j=1}^{n} \frac{\delta_{j}^{2}}{A_{j}}\left[e^{i k L_{j}} \widehat{q}_{0}^{(j)}(k)-e^{-i k L_{j}} \widehat{q}_{0}^{(j)}(-k)+2 k \sigma h_{0}^{(j)}(w, t)\right]+\sum_{r=n+1}^{n+m} \delta_{r}^{2} \widehat{q}_{0}^{(r)}(k)\right], \\
& A_{j}=e^{i k L_{j}}-e^{-i k L_{j}}, \quad B_{j}=e^{i k L_{j}}+e^{-i k L_{j}},(j=\overline{1, n}) . \\
&-418-
\end{aligned}
$$


The proof of the theorem is similar to given above theorems and can be obtained by combining previous results.

Authors are grateful to the reviewer for helpful comments and references which certainly helped to improve the paper.

\section{References}

[1] R.Albert, A.L.Barabasi, Statistical mechanics of complex networks, Rev. Mod. Phys. A, 74(2002), no. 1.

[2] R.Cohen, S.Havlin, Complex Networks: Structure, Robustness and Function, Cambridge University Press, 2010.

[3] T.Kottos, U.Smilansky, Periodic orbit theory and spectral statistics for quantum graphs, Ann. Phys., 274(1999), no. 1, 76-124.

[4] S.Gnutzmann, U.Smilansky, Quantum graphs: Applications to quantum chaos and universal spectral statistics, Advances in Physics, 55(2006), no. 5-6, 527-625.

[5] S.Gnutzmann, J.P.Keating, F.Piotet, Eigenfunction statistics on quantum graphs, Ann. Phys., 325(2010), 2595-2640.

[6] G.F.Dell'Antonio, E.Costa, Effective Schrödinger dynamics on o-thin Dirichlet waveguides via quantum graphs: I. Star-shaped graphs, J. Phys. A, Math. Theor., 43(2010), 474014.

[7] P.Exner, O.Post, A General Approximation of Quantum Graph Vertex Couplings by Scaled Schrödinger Operators on Thin Branched Manifolds, Commun. Math. Phys., 322(2013), $207-227$.

[8] H.Uecker, D.Grieser, Z.Sobirov, D.Babajanov, D.Matrasulov, Soliton transport in tubular networks: Transmission at vertices in the shrinking limit, Phys. Rev. E, 91(2015), 023209.

[9] G.Doetsch, Anleitung zum praktischen Gebrauch der Laplace-Transformation und der ZTransformation, Munchen, Wien: Oldenbourg, 1981.

[10] W.Rall, Branching Dendritic Trees and Motoneuron Membrane Resistivity, Experimental Neurology, 1(1959), no. 5, 491-527.

[11] R.R.Poznanski, Mathematical Neuroscience, San Diego, California, Academic Press, 2013.

[12] Z.A.Sobirov, M.I.Akhmedov, H.Uecker, Cauchy problem for the linearized KdV equation on general metric star graphs, Uzbek Mathematical Journal, 3(2015), 143-154.

[13] Z.A.Sobirov, D.Babajanov, D.Matrasulov, K.Nakamura, H.Uecker, Sine-Gordon solitons in networks: Scattering and transmission at vertices, EPL (Europhysics Letters), 115(2016), no. 5,50002 .

[14] D.Mugnolo, D.Noja, C.Seifert, Airy-type evolution equations on star graphs, Analysis \& $P D E, \mathbf{1 1}(2018)$, no. 7, 1625-1652.

[15] N.E.Sheils, D.A.Smith, Heat equation on a network using the Fokas method, J. Phys. A: Math. Theor., 48(2015), 335001.

[16] A.S.Fokas, A unified transform method for solving linear and certain nonlinear PDEs, Proc. Roy. Soc. A, 453(1997), 1411-1443. 
[17] A.Fokas, A Unified Approach to Boundary Value Problems, CBMS-NSF Regional Conference Series in Applied Mathematics, University of Cambridge, Cambridge, United Kingdom, 2008.

[18] N.E.Sheils, Multilayer diffusion in a composite medium with imperfect contact, Institute for Mathematics and Its Applications, arXiv:1610.02945v2 [math.NA] 21 Dec 2016.

[19] N.E.Sheils, Interface Problems using the Fokas Method, PhD Dissertation, University of Washington, 2015.

\section{Метод унифицированного преобразования (Фокса) для уравнения Шредингера на простом метрическом графе}

Гулмирза Худайберганов Зарифбой А. Собиров

Мардонбек Р. Эшимбетов

Национальный университет Узбекистана им. М. Улугбека

ВУЗ городок, Ташкент, 100174

Узбекистан

В настоящей работе мы получили интегральное представление решения начально-краевых задач для уравнения Шредингера в простых звездообразных графах методом Фокаса. Этот метод использует унифицированное преобразование Фуръе. Полученные нами решения дают более точную информацию о динамике рассеяния в точке разветвления графа. В частности, можно увидеть отдельные слагаемые интегрального представления, соответствующие отражению и прохождению волны через вершину графа.

Ключевые слова: уравнение Шредингера, метрический граф, разветвленные структуры, унифицированное преобразование, начально-краевая задача, преобразование Фуръе, краевая задача. 